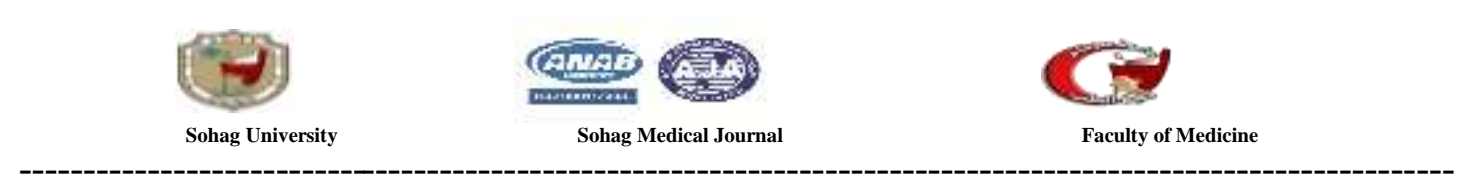

\title{
Foam sclerotherapy for reticular veins and telangiectasias
}

\begin{abstract}
Hossam Farouk Abdel-hamid, Professor of general surgery - general surgery department- Sohag faculty of medicine - Sohag university

Ahmed Saif Al Islam Abdalfatah , Assistant professor of vascular surgery vascular surgery department - Sohag faculty of medicine - Sohag university Kariem Ayman Mohamad Alsakaty, Senior resident at vascular surgery department - Sohag university hospitals
\end{abstract}

\begin{abstract}
Background Injection Sclerotherapy for the treatment of varicose veins in general and foam sclerotherapy for telangiectasias and reticular veins, in particular, has been used widely in the last decades. The procedure aims to occlude the lumen of varicose veins or superficial veins.

OBJECTIVE aiming to determine the safety profile by mean of a satisfactory complication rate and effectiveness in enhancing symptoms and limb appearance of foam sclerotherapy for the treatment of telangiectasias and reticular veins and to describe rates of procedure failure in terms of symptomatic or cosmetic varicose vein recurrence.

Search policy \& methods This study is a prospective study included 60 patients with telangiectasia and reticular veins in which assessment of side effect diversity and severity including the presence of cardiovascular or pulmonary events and the degree of injected vein disappearance and patient satisfaction as outcomes.

Results 60 patients included in this study between them were 9 males (15\%) 51 females (85\%), The rate of adverse events was relatively small for all recorded complications including hyperpigmentation $(20 \%)$, skin ulceration $(6.7 \%)$, pain after sclerosant injection $(5 \%)$, and matting and /or new vessel formation (3.3\%). The net improvement percentage was $91.7 \%$ represent the disappearance of injected veins and success of procedure with $8.3 \%$ of cases show no improvement. No major adverse events occurred.

Conclusion Foam sclerotherapy is safe, easy and efficient for the treatment of leg varicosities, reticular veins, and telangiectasias. major adverse events are rare.
\end{abstract}

Keywords: foam sclerotherapy, telangiectasias, reticular veins

\section{Introduction}

Sclerotherapy includes the injection of sclerosant in both a liquid and a foam form, which adheres to the endothelial vessel lining, causing a thrombophlebitis like reaction. This process results in fibrosis of injected veins as a treatment of telangiectasias and reticular veins.[1] sclerotherapy is considered the principle standard When performed correctly, sclerotherapy can harvest excellent outcomes with an acceptable side effect profile. Improvement of $80 \%$ to $90 \%$ is usually achieved.[2]

Sclerotherapy can be described the chemical ablation of varicose veins by intravenous injection of foam or liquid sclerosant in which the injected veins may be located intradermal, subcutaneous, and or trans-facial.[3] The definition of a sclerosing foam (SF) is a combination of gas and liquid sclerosing solution (detergent type) with tension- 
active nature. The gas have to be well tolerated or physiologic and the bubble size less than 100 micros.[4]

The optimal sclerosing agent should exhibit few adverse effects, including injection site hyperpigmentation, skin ulceration, matting of telangiectatic veins or neovascularization, and probable allergic hypersensitivity. This ideal sclerosing agent should have maximal clinical efficiency with a low number of injection sessions needed to produce the anticipated clinical results. it also must be associated with minimal patient discomfort Foam sclerotherapy considered safe and effective treatment options in treating telangiectasias and reticular veins.[5]

\section{Patient and methods}

This was a prospective study of 60 undergoing sclerotherapy. All patients was seeking for receiving treatment of telangiectasias and reticular veins. The majority of patients received treatment with polidocanol solution in the foam form for reticular veins and less concentrated foam solution for telangiectasias

\section{Inclusion criteria}

Adult patients more than 16 years old with symptomatic or cosmetic varicosities, telangiectasia, and reticular veins. - Residual reticular veins or telangiectasia after previous intervention. No evidence of limb ischemia. Normal values blood tests.

\section{Exclusion criteria}

- Patients complaining of acute superficial thrombophlebitis. - Patients at great risk of DVT such as patients with hypercoagulability state and active cancer. - Patients with a previous history of DVT and or pulmonary embolism. Patients who are females with pregnancy. - Patients with allergy to the sclerosing agents used. - Patients with local infection in the area targeted for injection sclerotherapy. - Patients with severe generalized infection and other skin diseases or lesions. Patients with longstanding immobility or prolonged confinement to bed. - Patients with peripheral arterial insufficiency (chronic limb ischemia). - Diabetic patients with severe peripheral neuropathy. - Patients requiring continuous use of oral corticosteroids. - Patients with clotting disorders.

\section{Methods}

\section{1- Preparation of the patient}

- The patient was placed supine assuring Good illumination. Then the skin was smeared with alcohol to make the telangiectasia easily visible through the glistening effect of the alcohol also makes the skin more transparent and helps to disinfect the injection site.

\section{2- Preparation of the sclerosant foam}

- In our study, we used the Tessari technique for the purpose of generating foam from sclerosant, which mainly was polidocanol (1-3\%). This technique is accepted and used methods in foam sclerotherapy production. We used two disposable syringes and a three-way stopcock or a two-way female-female Luer-lock connector. - Under complete aseptic precautions the two syringes were connected using the three-way tap. $10 \mathrm{ml}$ syringes were used. Then a mixture of sclerosant and air was drained into one syringe at a percentage of one part of sclerosing material to four parts of gas. The mixture wavered forcefully between the two syringes about 10 to 20 times. The produced foam in this way was stable for about 2 minutes therefore the foam should be used as soon as possible it prepared.

\section{3- Application of the sclerosing foam}

- Targeted telangiectasias and reticular veins were injected with the sclerosing foam using a direct puncture technique. By using 30 or $25 \mathrm{~g}$ needle with the bevel pointing upward superficial varices were injected first, $1 \mathrm{ml}$ was 


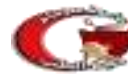

Faculty of Medicine

injected per injection site and the foam was disseminated by massaging the leg with the hand. The injection was slow and steady beginning injection from largest veins and then into small veins, which means that Treatment proceeded from the most proximal targeted veins to the most distal ones starting injection in the most complaining areas to the patient.

-After the injection sclerotherapy session, patients kept in supine position for 5 minutes and the injected areas were compressed with a piece of gauze and Steady external compression is applied over the whole leg. Then the leg with injected areas is bandaged for at least 7 days with a maximum duration of 14 days. After end of the procedure patients were advised of Immediate ambulant and return to normal activity.

Data analysis Collected data was statistically analyzed Data were analyzed using SPSS version 23. Charts and graphs were produced using either SPSS or Excel. The P-value was considered significant if it was less than 0.05 .

\section{Results}

- 60 patients were incorporated in this study of which fifty-one 51 patients were females $(85 \%)$ and nine 9 males $(15 \%)$. the age of the patients ranges from 18 to 41 years with a mean of $29.57( \pm 5.791)$ years.

Table 1: Shows success of the procedure in terms of disappearance of the injected veins

\begin{tabular}{|r|r|}
\hline $\begin{array}{r}\text { Disappearance of } \\
\text { injected veins }\end{array}$ & frequency \\
\hline Dis disappeared (succe & $55(91.7 \%)$ \\
\hline $\begin{array}{r}\text { not disappeared } \\
\text { (failed) }\end{array}$ & $5(8.3 \%)$ \\
\hline
\end{tabular}

Table 2: shows relation between gender and success rate

\begin{tabular}{|l|r|r|r|}
\hline \multicolumn{1}{|c|}{ Success of treatment } & male & Female & P value \\
\hline $\begin{array}{l}\text { Disappeared injected } \\
\text { veins }\end{array}$ & $7(77.8 \%$ & $4 .(98.1 \%)$ & .102 \\
\hline $\begin{array}{l}\text { NotDisappeared } \\
\text { injected veins }\end{array}$ & $2(22.2 \%$ & $3(5.9 \%)$ & \\
\hline
\end{tabular}

- No statistically significant correlation between gender and disappearance of injected veins $(p=.102)$

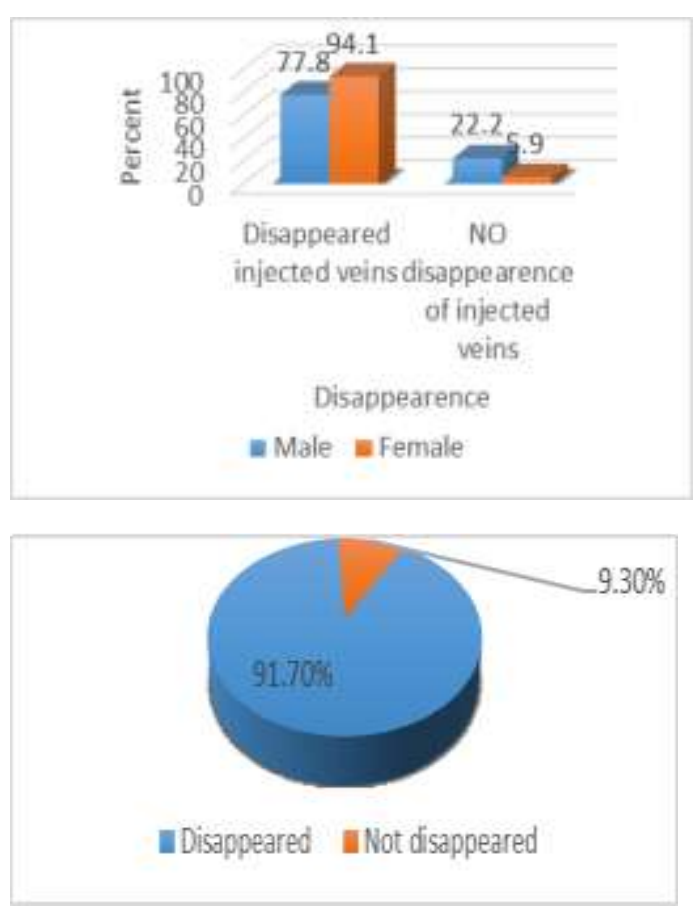

\section{Discussion}

CVD is a worldwide problem, varicose veins disturbing more than 25 million adults in the United States with more than 6 million having one degree or more of advanced venous disease. Sclerotherapy is thought to be the typical treatment for superficial intra-cutaneous varicose veins like telangiectasias, spider veins and reticular veins, producing an enhancement of up to $80 \%$ or $90 \%$ to be attained. Using a sclerosing foam it is a must to consider that the sclerosing agent in this foam form has a prolonged contact time with the injected vein and is more active hence a lower amount and lower concentration of the sclerosing agent are to be used compared with sclerosing agent in the liquid form.[6] Foam sclerotherapy could 
be used for both large varicose veins and spider veins. For sclerotherapy of spider veins reticular veins and telangiectasias, low concentrations of sclerosant like polidocanol are needed or, the inflammatory reaction will be more pronounced. one should keep in mind that in these small veins sclerotherapy with liquid polidocanol is very efficient and safe.[7] Our study includes 60 sixty patients with telangiectasia and or reticular veins. Away much like Robert L in his study 2005 depends on several studies reporting the results of sclerotherapy in small series of patients. [8]

In our study $52(86.7 \%)$ of patients presented seeking for cosmetic issues, within them were 48 (92.3\%) females, this female gender predominance may be explained by the more seeking for cosmetic problems including telangiectatic veins by females more than males the same was noticed by Zimmet and his colleagues in 2003.[9]

In our study cosmetic issues were the main presenting symptom (86.7\%) pain was the second main presenting symptoms by $(78.3 \%)$ followed by pruritis $36.7 \%$ then bleeding varix $(6.7 \%)$. while Nael et al reported pain to be the most common presenting symptoms $(81 \%)$ pruritis $(41 \%)$ varix rupture (3\%).[4]

Regarding complications and risks, it must be noticed that not all sclerosing agents cause contact skin necrosis at certain concentrations when injected outside veins. Even polidocanol in concentrations between 0.25 and $1 \%$ may not cause skin necrosis or ulceration by accidental paravenous injection of moderate quantities. In our study (6.7\%) of cases had skin necrosis after paravenous extravasation Nael et al in their study had reported (1\%) skin necrosis after paravenous extravasation while palm and his colleagues had (1.3\%).[10] in our study Twelve patients $(20 \%)$ have some degree of hyperpigmentation after injection of foam not much far from results obtained by Nael et al (23\%) and comparable to that obtained by Palm et al (17.8\%).[4] According to Wright D.D occurrence of skin Hyperpigmentation and neovascularization are the most commonLocal complication of sclerotherapy, the incidence of temporary hyperpigmentation in our study ranges from 10 to $30 \%$. Telangiectatic matting (new vein formation) happened in two cases $(3.3 \%)$ in our study comparable to $(8 \%)$ that reported by Palm et al. There is no agreement about protocols for the treatment of adverse events such as matting although laser therapy may be used.[11]

In our study, major side effects were low (1.7\%), compared to the study in 2010 done by Palm, Guhia and Goldman which assessed the total occurrence of complications after foam sclerotherapy to below, $(0 \%$ to $5.7 \%)$ of patients. Postprocedure care may clarify to some extent the low incidence of occurrence of side effects in our study. We asked the patients to wear class II $(30-40 \quad \mathrm{mmHg})$ compression stockings for 7 days, 24 hours a day, or compression bandage along the whole injected lower limb after treatment and instruct frequent ambulation in the weeks after treatment.[4]

\section{Conclusion}

Foam sclerotherapy appears safe, effective with no major adverse effects in the treatment of telangiectasia, and reticular veins. Foam sclerotherapy is not without risks pain, thrombophlebitis, skin hyperpigmentation, and deep venous thrombosis DVT are possible complications may result but in general, serious or major adverse effects are rare. Therefore, with proper patient selection, adherence to the injection protocols foam sclerotherapy is safe effective method of treatment telangiectasia and reticular veins. 


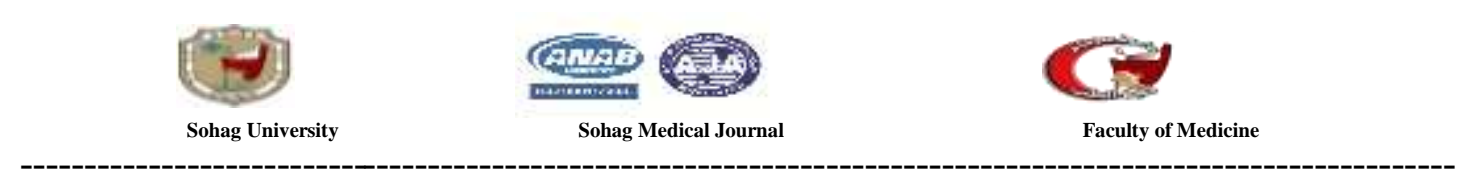

\section{References}

1. Scovell, S. and J.S. Dover, Liquid, foam, and glue sclerotherapy techniques for the treatment of lower extremity veins. UpToDate, Collins, KA (Ed), UpToDate, Waltham, MA. Accessed, 2018. 4: p. 17.

2. Thomson, L., Sclerotherapy of telangiectasias or spider veins in the lower limb: a review. Journal of Vascular Nursing, 2016. 34(2): p. 61-62.

3. Gibson, K. and K. Gunderson, Liquid and Foam Sclerotherapy for Spider and Varicose Veins. Surgical Clinics, 2018. 98(2): p. 415-429.

4. Nael, R. and S. Rathbun ' Effectiveness of foam sclerotherapy for the treatment of varicose veins. Vascular Medicine, 2010. 15(1): p. 27-32.

5. Kugler, N.W. and K.R. Brown, An update on the currently available nonthermal ablative options in the management of superficial venous disease. Journal of Vascular Surgery: Venous and Lymphatic Disorders, 2017. 5(3): p. 422-429.
6 Halvorson, C.R., R.A. Weiss, and M.A. Weiss, Therapy of Telangiectasia and Varicose Veins and Their Complications. Textbook of Cosmetic Dermatology, 2017. 16: p.312 .

7. Napoli, S., et al., Sclerotherapy, in European Handbook of Dermatological Treatments. 2015, Springer. p. 1307-1312.

8. Worthington-Kirsch, R.L. Injection sclerotherapy. in Seminars in interventional radiology. 2005. Thieme Medical Publishers.

9. Zimmet, S.E., Sclerotherapy treatment of telangiectasias and varicose veins. Techniques in Vascular \& Interventional Radiology, 2003. 6(3): p. 116-120.

10. Palm, M.D., I.C. Guiha, and M.P. Goldman, Foam sclerotherapy for reticular veins and nontruncal varicose veins of the legs: a retrospective review of outcomes and adverse effects. Dermatologic Surgery, 2010. 36(s2): p. 1026-1033.

11. Wright, D.D. What is the current role of foam sclerotherapy in treating reflux and varicosities? in Seminars in vascular surgery. 2010. Elsevier. 